\title{
La couverture des risques en aquaculture. Une réflexion sur le cas de la conchyliculture en France
}

Risks hedging in aquaculture. A note on of shellfish farming in France

\section{Véronique Le Bihan et Sophie Pardo}

\section{(2) OpenEdition}

Journals

Édition électronique

URL : http://journals.openedition.org/economierurale/3419

DOI : $10.4000 /$ economierurale.3419

ISSN : 2105-2581

Éditeur

Société Française d'Économie Rurale (SFER)

\section{Édition imprimée}

Date de publication : 30 mai 2012

Pagination : 16-32

ISSN : 0013-0559

\section{Référence électronique}

Véronique Le Bihan et Sophie Pardo, « La couverture des risques en aquaculture. Une réflexion sur le cas de la conchyliculture en France », Économie rurale [En ligne], 329 | mai-juin 2012, mis en ligne le 30 mai 2014, consulté le 19 avril 2019. URL : http://journals.openedition.org/economierurale/3419 ; DOI : 10.4000/economierurale.3419 


\section{La couverture des risques en aquaculture Une réflexion sur le cas de la conchyliculture en France}

Véronique LE BIHAN et Sophie PARDO • Laboratoire d'économie et de management de NantesAtlantique, Lemna, Université de Nantes

a conchyliculture, qui regroupe les acti-vités d'élevage des huîtres, des moules, des coques et des palourdes, est une activité structurante du littoral français. Cette activité est très fortement exposée aux risques, notamment climatiques et sanitaires donnant lieu à des interdictions de commercialisation fréquentes, et a connu des crises majeures conduisant à l'extinction d'espèces (huîtres portugaises) ou à la restructuration d'activités économiques (vénériculture ${ }^{1}$ ).

Lors d'une étude sur la gestion globale des risques en conchyliculture (Le Bihan et al., 2007), les conchyliculteurs soulignent que les solutions existantes en matière de couverture de risques ne répondent pas à certains de leurs besoins, notamment concernant trois risques liés au milieu de production : les fermetures sanitaires, les mortalités cheptel et les aléas climatiques. Depuis 2007, les professionnels font face à une augmentation du nombre de sinistres. En effet, les épisodes de surmortalités estivales de 2008 à 2011, la tempête Xynthia qui a touché la côte Atlantique française en 2010 et de nombreuses interdictions de commercialisation, fragilisent ce secteur et accentuent la demande des professionnels en matière de couverture des risques.

La gestion des risques conchylicoles se décline majoritairement en mesure de prévention et d'auto-assurance et systèmes de solidarité. Aujourd'hui, il n'existe que très peu de polices d'assurance disponibles pour les risques liés au milieu de production. En particulier, aucune assurance couvrant les

1. La vénériculture est l'élevage de la palourde. À la suite de la maladie des anneaux bruns en 1987, la mono-production en vénériculture a disparu (Vigo, 2001). risques de maladie ou de mortalité des coquillages n'est disponible sur le marché. Ce constat pose la question de la définition et de l'assurabilité de tels risques.

Les facteurs d'inassurabilité ont été étudiés par Gollier (1996). Il souligne notamment l'importance de l'aléa moral et de l'ambiguïté qui porte sur certains risques. Cette question a fait également l'objet de travaux concernant les risques catastrophiques (Zeckhauser, 1995) et les catastrophes naturelles en particulier (Jaffee, Russell, 1997). Dans ce cas précis, des solutions de marché (obligations catastrophes, contrats d'option) ont été mises en place afin de surmonter certains freins liés à la limite de capacité financière du secteur assurantiel face à l'ampleur potentielle des dommages. En revanche, peu de travaux traitent de la couverture des risques en conchyliculture. À notre connaissance, seuls Beach et Viator (2008) abordent cette question. Ils étudient un programme pilote d'assurance pour les clams aux USA et mettent en évidence les difficultés rencontrées dans la mesure des pertes et la détermination des causes des sinistres. Dans le secteur agricole au contraire, une littérature abondante traite de la question des risques et de leur couverture, tant du point de vue théorique qu'empirique. En particulier, Couture (2009) traite d'une problématique similaire à la nôtre dans le secteur forestier. Il analyse les principaux risques auxquels font face les forestiers et met en évidence les difficultés liées à la mise en place d'un système d'assurance des risques naturels efficace.

Dans un contexte d'augmentation des sinistres et face à l'incomplétude des instruments de couverture, cet article propose 
RECHERCHES

Véronique LE BIHAN, Sophie PARDO

Tableau 1. Principales crises enregistrées par le secteur conchylicole

\begin{tabular}{l|l}
\hline Période & \multicolumn{1}{c}{ Événements } \\
\hline 1920 & Mortalité massive de l'huître plate (Bretagne) \\
\hline 1971 & Disparition des huîtres portugaises (National) \\
\hline Années 1970 & Forte baisse de productivité des bassins ostréicoles (National) \\
\hline 1987 & Épizootie d'origine bactérienne de la palourde (National) \\
\hline 1978,1999 et 2003 & $\begin{array}{l}\text { Pollutions par hydrocarbure Amoco Cadiz, Erika et Prestige (Bretagne, Pays de la Loire, } \\
\text { Poitou-Charentes, Aquitaine) }\end{array}$ \\
\hline 2008 et 2009 & Fortes mortalités inexpliquées des huîtres juvéniles (National) \\
\hline 1999 et 2010 & Tempêtes Lothar et Xynthia (Régions côte atlantique) \\
\hline
\end{tabular}

Source : les auteures

une première réflexion sur les risques en conchyliculture, les outils de gestion disponibles et les difficultés auxquelles se heurte le développement de nouveaux instruments. Dans un premier temps, nous mettons en évidence la spécificité des risques conchylicoles et nous proposons une typologie adaptée. Dans un deuxième temps, nous étudions les méthodes de prévention et d'auto-assurance mises en œuvre par les professionnels, en soulignant les liens avec les obligations réglementaires. À l'aide d'un état des lieux des instruments de couverture disponibles pour les conchyliculteurs, nous montrons la prédominance des systèmes d'intervention publique et la faible étendue des contrats d'assurance privée. Les limites des mesures mises en œuvre par les professionnels et les freins au développement assurantiel, sont étudiés dans un troisième temps. En particulier, nous analysons les éléments à l'origine de l'inassurabilité de certains risques liés au milieu de production. Nous montrons que la définition des risques sanitaires et la quantification des dommages sont délicates, qu'il existe une forte ambiguité pour les risques liés au milieu de production et que l'étroitesse du secteur contribue fortement à des coûts de transaction élevés. Ce travail préliminaire est essentiel pour identifier les pistes de travail futures en matière de couverture des risques.

\section{Définition et typologie des risques conchylicoles}

La conchyliculture est l'une des activités historiques et structurantes du littoral français. Bien que s'exerçant en mer, le caractère agricole de l'aquaculture marine a été reconnu par la Loi d'orientation sur la pêche maritime et les cultures marines de 1997. Cette classification consolide et élargit l'accès des aquaculteurs marins à plusieurs dispositifs d'aides existant en agriculture. Le secteur conchylicole est relativement étroit ; il représente 3244 entreprises, essentiellement familiales, pour 19168 emplois salariés ou non. Ce secteur se concentre : $13 \%$ d'entreprises en moins entre 2001 et 2006. Ce phénomène s'explique en partie par un renouvellement difficile des entreprises conchylicoles françaises (Le Bihan, 2006), les départs à la retraite (génération du babyboom) n'étant pas compensés par les installations. Les métiers de la conchyliculture sont en effet jugés pénibles par les jeunes avec des contreparties financières insuffisantes. L'ensemble des surfaces conchylicoles permet de produire environ 193600 tonnes de coquillages pour un chiffre d'affaires estimé à 400 millions d'euros ${ }^{2}$ en 2007. Représentant un peu plus des deux tiers de la production totale conchylicole française, l'ostréiculture domine ce secteur, suivie de la mytiliculture (30\%).

L'histoire de la conchyliculture française est rythmée par des crises (tableau 1) plus

2. Source MAP-DPMA, 2009. 
ou moins profondes liées au milieu d'élevage des coquillages. Compte tenu de leurs conséquences pluriannuelles, certaines crises ont profondément marqué la profession et ont connu un retentissement médiatique important.

Au-delà de ces évènements qui ont affecté l'ensemble de la filière, les conchyliculteurs doivent faire face à des sinistres récurrents, géographiquement localisés. La présence de phycotoxines dans les coquillages, dangereuses pour la consommation humaine, entraîne ainsi des interdictions de commercialisation fréquentes, de durées plus ou moins longues, dans des zones de production telles que le bassin d'Arcachon ou l'étang de Thau.

Si les risques liés à l'environnement de production sont prépondérants, les entreprises conchylicoles sont également exposées à d'autres types de risques inhérents aux activités primaires agricoles. Hardaker et al. (2004) proposent une classification des risques agricoles en 5 groupes : le risque de production (résultant d'aléas climatiques, technologiques, sanitaires ou épidémiques) ; le risque de prix ou de marché ; le risque institutionnel (changements de politique ou de régulation); les risques de personnes; le risque financier (de taux, de défaut, de liquidité). Cette classification peut s'appliquer au secteur des cultures marines, composante du secteur agricole, mais elle ne permet pas d'appréhender précisément le poids de certains risques spécifiques au secteur. Nous proposons donc une nouvelle typologie synthétisée dans la figure 1 , avec quatre grandes catégories de risques qui couvrent l'amont à l'aval de la filière conchylicole : les risques liés au milieu de production comprenant les risques sanitaires, climatiques et de pollution ; ceux liés à l'environnement de l'entreprise et aux instruments nécessaires à la production ; ceux liés aux produits et aux débouchés et ceux relevant de l'environnement institutionnel. Certains d'entre eux ne diffèrent pas des risques d'autres secteurs agricoles (les risques liés à la commercialisation et à la distribution, et une partie des risques liés à l'entreprise). Aussi, nous ne les détaillerons pas et nous ne présenterons que les risques qui témoignent des spécificités du secteur conchylicole.

\section{Les risques liés au milieu de production}

Les risques de production sont exacerbés car les activités conchylicoles s'exercent en milieu ouvert. Parmi les grandes crises du secteur, on a enregistré trois pollutions par hydrocarbure en moins de 30 ans (tableau 1). Ces évènements ont eu des conséquences très importantes sur l'environnement et sur la filière, notamment en termes d'image. D'autres types de pollution touchent le milieu de production, en particulier celles liées aux rejets agricoles. Selon Bommelaer et Devaux (2011), 37 \% des zones conchylicoles bretonnes déclassées entre 2000 et 2010, l'ont été à cause de la dégradation de la qualité des eaux côtières liée aux rejets.

Le milieu de production induit une variabilité des quantités de coquillages mises en élevage impactant le rendement. La production de coquillages est essentiellement dépendante du recrutement naturel qui dépend lui-même des facteurs environnementaux (hydrobiologie, climatologie, courantologie). La croissance des coquillages est entièrement tributaire de la ressource trophique, les professionnels ne pouvant intervenir pour compenser un déficit nutritif contrairement aux agriculteurs. Enfin, les interactions fortes intra et inter bassins sont à l'origine d'une exposition plus intense des cheptels aux risques sanitaires ou épidémiologiques. Les transmissions des épizooties peuvent ainsi être facilitées par les courants marins. Si certains traitements préventifs ou curatifs existent en agriculture, les conchyliculteurs ne peuvent y recourir et sont entièrement dépendants des aléas climatiques et sanitaires. 
Les principaux aléas climatiques touchant le secteur sont les tempêtes et submersions marines. Ces catastrophes naturelles demeurent rares mais affectent à la fois les installations à terre et les cheptels en mer. Dans le contexte du réchauffement climatique, il est admis que la fréquence des aléas climatiques va augmenter, il est donc à craindre un accroissement de l'exposition des conchyliculteurs à ces phénomènes.

Le poids des risques sanitaires est extrêmement important dans le secteur. En effet, outre les crises qui ont touché l'ensemble du secteur (tableau 1), les conchyliculteurs sont confrontés à des phénomènes plus récurrents tels que la présence de phycotoxines dangereuses pour la santé humaine ou pour la faune marine. En conséquence, le nombre de jours par an d'interdiction administrative de vente et de ramassage de coquillages a été multiplié par 5 entre 1984 et 2003 (Belin, 2005). Par ailleurs, depuis 2008, l'ensemble des élevages ostréicoles français est touché l'été par une épizootie qui détruit les juvéniles d'huîtres. Auparavant, des cas de surmortalités estivales de jeunes huîtres avaient déjà été constatés dans certains bassins, lors de hausse des températures de surface (Maurer et al., 1986 ; Samain, McCombie, 2007). Mais le phénomène apparu chaque été depuis 2008 est d'une toute autre ampleur, avec un taux de mortalité atteignant 40 à $100 \%$ des stocks en élevage (Cochennec-Laureau, Baud, 2011). La cause la plus souvent invoquée est désormais celle d'un virus mutant (OsHV$1 \mu$ Var) qui devient actif à partir de températures plus basses que celles habituellement constatées pour ce type de virus (16-17 ${ }^{\circ} \mathrm{C}$ au lieu de $19{ }^{\circ} \mathrm{C}$ ); ce facteur reste donc en partie lié à des conditions climatiques.

Il faut souligner que dans le contexte du réchauffement climatique, l'évolution des aléas naturels, demeure incertaine et représente un facteur aggravant pour les risques sanitaires. Les incidences de la hausse de température prévue, restent en grande partie indéterminées (Arnason, 2007) : une décalcification des coquillages due à l'acidification des océans (Pörtner, 2008 ; Hallegatte et al., 2007), un surcaptage de naissain, un développement des virus et parasites pour les coquillages (Gitay et al., 2002), etc. Les conséquences pour les organismes marins sont encore largement méconnues, bien que les juvéniles et les larves semblent afficher une plus grande sensibilité au phénomène (Vézina, Hoegh-Guldberg, 2008).

Enfin, l'incidence des comportements des producteurs entre eux et plus particulièrement l'existence d'externalités négatives réciproques est également une caractéristique spécifique au secteur conchylicole. Au sein d'un même bassin, les cheptels mis en élevage par différents producteurs, partagent une ressource trophique fluctuante. Son renouvellement dépend des marées, des caractéristiques du milieu et des conditions climatiques. Les intensités de charge des parcs peuvent être à l'origine d'une baisse de productivité de l'ensemble du bassin ou être un vecteur favorisant les épizooties. En effet, les schémas des structures régulant l'intensité de production par bassin, ne permettent pas toujours de mettre en adéquation les niveaux de stocks de coquillages et les nutriments nécessaires à leur croissance car ils ne spécifient pas le nombre de bêtes par hectare.

\section{Les risques liés à l'entreprise}

Les entreprises conchylicoles sont peu sensibles au risque de prix ou de marché comparativement aux entreprises agricoles. Hormis pour certains professionnels très spécialisés, le poids des intrants est faible comparativement à celui constaté dans les secteurs agricoles. Les professionnels ne subissent pas non plus de fortes variations des prix de marché. Plusieurs raisons à cela : - l'effet de la concurrence internationale sur les huîtres est négligeable (la France produit $87 \%$ des huîtres européennes) ${ }^{3}$.

3. Source Fishstat FAO, 2008. 
Concernant les moules, le décalage de saison commerciale permet le positionnement des produits nationaux en dehors des périodes de commercialisation des produits étrangers ; - les cycles pluriannuels des coquillages ( 3 ans pour produire une huître, 2 ans pour produire une moule), les multiples techniques d'élevage, les écloseries (pour le naissain d'huîtres) et les différentes zones de production des producteurs permettent de lisser pour partie les quantités mises en marché assurant ainsi une certaine stabilité des prix. Les prix négociés en début de saison entre les grands courtiers et les producteurs sont également un facteur atténuant de la fluctuation des cours.

Le faible impact des risques de marché sur ces entreprises est un élément très important qui explique, en partie, une relative résistance des entreprises conchylicoles (moins de $2 \%$ de faillite selon le Bureau de la direction départementale des territoires et de la mer de Charente-Maritime) malgré la forte exposition aux risques naturels. Une crise majeure peut néanmoins remettre en question cet équilibre. C'est le cas des surmortalités de 2008 à 2011 qui, en décimant le stock des juvéniles trois années consécutives, ont supprimé la possibilité de lissage de la production de chaque éleveur et remettent en cause la stabilité des prix du marché.

La dernière spécificité associe les risques financiers et institutionnels et a trait au statut juridique des concessions. Le régime des concessions conchylicoles se situe à la frontière du domaine public et du domaine privé. Les conchyliculteurs exercent ainsi une activité sur le Domaine public maritime (DPM), lequel est géré par l'État dans l'intérêt de la Nation. Cet espace est donc inaliénable et imprescriptible. Si une certaine stabilité est observée quant aux attributions ou renouvellements des concessions, ces autorisations sont de plus en plus aisément révocables en raison notamment des pressions grandissantes d'autres usages du littoral (pêche, tourisme). Mongruel et al. (2006) montrent qu'il existe néanmoins un phénomène de patrimonialisation du droit $d$ 'accès à la ressource primaire et du droit d'exploitation, dans la mesure où il est possible en pratique de leur attribuer une valeur et de les monnayer lors de la cession des parcelles entre conchyliculteurs. Ces valeurs constituent la part la plus importante dans la valorisation de l'entreprise exposant le professionnel à un risque financier important lors de la cession. Enfin, le caractère inaliénable du DPM interdit son nantissement ou son hypothèque et pose parfois des problèmes en termes de garantie lors de négociations avec les financeurs. Cette difficulté s'impose plus particulièrement pour les jeunes qui souhaitent s'installer et qui ne disposent pas d'apport en capital.

\section{Les risques institutionnels}

À l'instar des agriculteurs, les conchyliculteurs font face à des changements de réglementation. Deux éléments spécifiques distinguent néanmoins leur exposition aux risques institutionnels.

Le premier est lié à la nature des produits élevés et à leur type de consommation, la commercialisation des coquillages et leur consommation s'effectuant avec des produits vivants. Cette mise en marché induit de très nombreux contrôles afin de proposer des coquillages. Dans un contexte d'affermissement des normes de sécurité alimentaire et de recours croissant au principe de précaution (Collart-Dutilleul, 2003), le cadre réglementaire est contraignant. Cela se traduit par une augmentation du nombre de jours d'interdiction de ventes de coquillages qui peut entraîner des préjudices conséquents pour les entreprises (pertes de clients, insuffisance de trésorerie).

Le second élément tient aux conditions d'attribution des parcs et limite la flexibilité des entreprises au sein du secteur conchylicole. À chaque concession est attribuée une espèce de coquillages (plus rarement deux ou trois espèces) et une technique d'élevage. Aussi, un professionnel qui souhaite- 
RECHERCHES

Véronique LE BIHAN, Sophie PARDO

Figure 1. Risques, conséquences et gestion des risques en conchyliculture

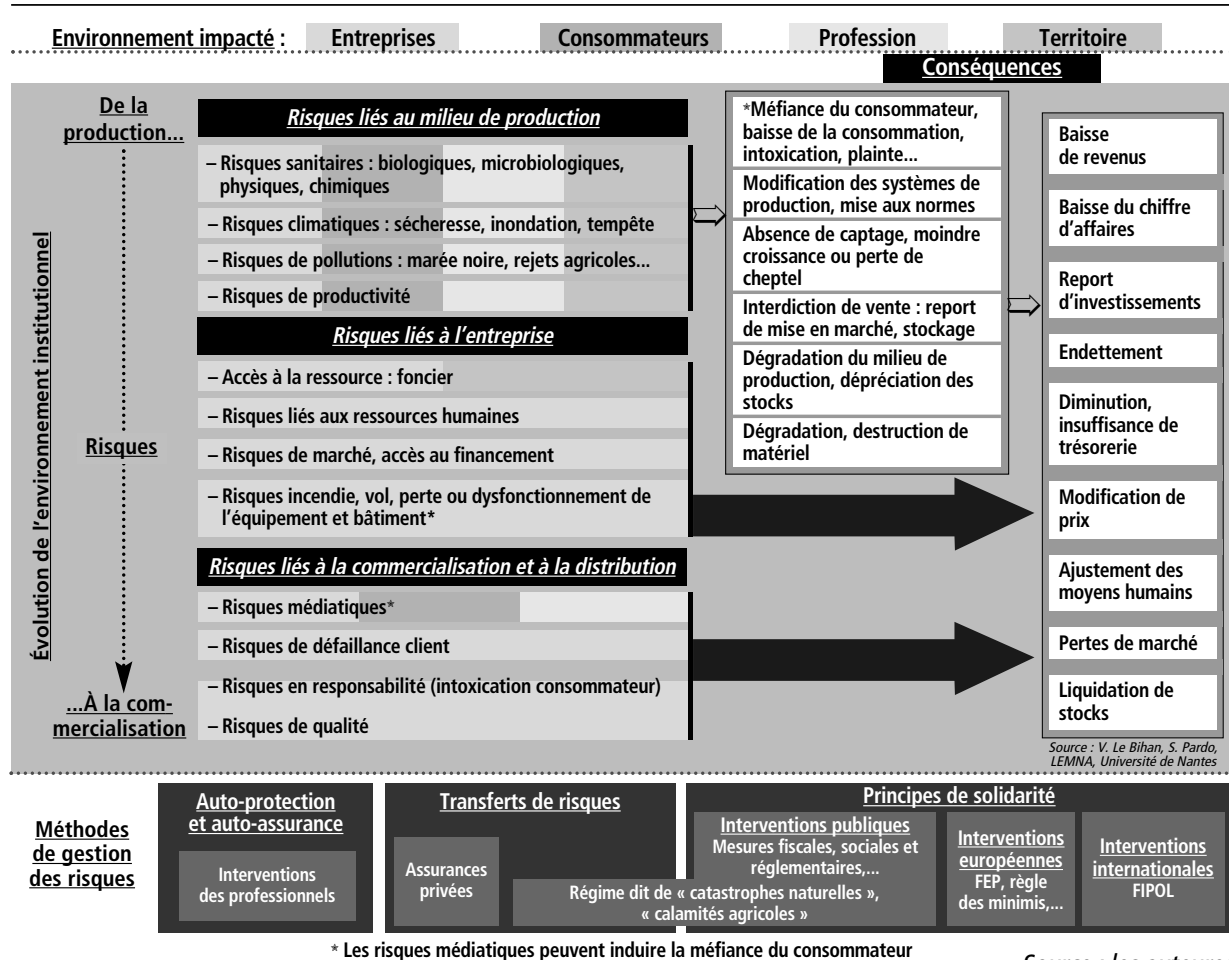

Source : les auteures

rait changer de culture ou de méthode d'élevage sur ses parcs, pour compenser une rentabilité moindre, doit en faire la demande à l'autorité de gestion des concessions. Dans les faits, ces autorisations sont très difficiles à obtenir et supposent des délais importants.

De la production à la commercialisation, les conséquences de ces risques peuvent être multiples, directes ou induites (figure 1). Certains risques sont de type catastrophique. Si la réalisation de ces risques est indépendante des activités des conchyliculteurs, leurs pratiques influencent néanmoins l'ampleur des conséquences. En outre, la portée géographique de certains préjudices peut varier du bassin local au territoire national. Au niveau microéconomique, les préjudices majeurs se traduisent financièrement par des diminutions de trésorerie, des reports d'investissements. Les conséquences peuvent néanmoins être très différentes selon les entreprises en fonction de leurs métiers, de leurs caractéristiques technicoéconomiques et de leur implantation géographique.

\section{Les couvertures de risques conchylicoles}

Les besoins des professionnels en matière de couverture de risques se concentrent naturellement sur les risques liés au milieu de production (Le Bihan et al., op. cit.). Comme l'illustre la figure 1, les méthodes de gestion de ces risques relèvent à la fois de la prévention et d'assurances privées mais aussi de l'intervention publique et de mécanismes de solidarité, dont on peut souligner la prédominance. Cependant, les couvertures disponibles aujourd'hui sont loin d'être suffisantes, en particulier pour les risques sanitaires. Dans cette section, nous présentons un panorama des outils 
existants en matière de couverture des risques liés au milieu de production.

\section{Auto-protection et auto-assurance}

Le premier principe de gestion des risques concerne le recours à des modes de prévention et de couverture individuels de la part des conchyliculteurs. Ehrlich et Becker (1972) définissent l'auto-protection comme les actions entreprises pour réduire la probabilité de sinistre alors que l'auto-assurance recouvre les actions limitant le coût des pertes à la suite d'un sinistre. La dépendance au milieu naturel a conduit de tout temps les professionnels à s'adapter et à intégrer les risques dans leur pratique. Ainsi, leurs choix technico-économiques se modifient constamment pour adapter de manière efficace l'outil de production aux conditions naturelles et réduire l'exposition de l'entreprise aux aléas.

Dans le cadre de l'auto-protection contre les risques liés au milieu de production, les conchyliculteurs optent pour une diversification qui peut revêtir plusieurs formes : production d'espèces différentes (Gervasoni et al., 2011), activités complémentaires (pêche, tourisme bleu). La diversification des sources d'approvisionnement de naissain (propre captage ou achat de naissain naturel, naissain d'écloserie) est également un moyen pour lisser les quantités mises en élevage. Les ostréiculteurs, à travers l'élevage d'huîtres de ploïdies ${ }^{4}$ différentes ont la possibilité de modifier la durée d'élevage (raccourcissement des phases de croissance pour les huîtres triploïdes) mais également la saisonnalité des ventes. La diversification peut également concerner les espaces géographiques. Les entreprises disposant de concessions dans diverses zones de production peuvent bénéficier d'espaces moins exposés aux fermetures sanitaires ou d'une meilleure productivité naturelle. De plus, certains producteurs délocalisent une

4. La ploïdie d'une cellule caractérise le nombre d'exemplaire de ses chromosomes (Universalis). partie de leur production à l'étranger (Irlande et Maroc par exemple).

Les démarches de prévention des risques, imposées par des normes réglementaires, sont nombreuses et concernent aussi bien les outils de production que les coquillages. Leur application participe ainsi aux mesures d'auto-protection contre les risques sanitaires. Ainsi, le paquet hygiène ${ }^{5}$ formalise les procédures de traçabilité et de sécurité sanitaire des aliments. Des démarches collectives sont également développées via les Schémas de mise en valeur de la mer (SMVM) ou les contrats de baie ou d'étang avec l'agence de l'eau. Outils privilégiés de la gestion du littoral et des plans d'eau, ces différents outils précisent la vocation des espaces et assurent la cohérence entre ses différents usages notamment entre la protection de l'environnement et le développement économique. Ces différentes normes et programmes collectifs contribuent à diminuer les probabilités d'occurrence d'un certain nombre de risques sanitaires.

La première mesure importante d'autoassurance contre les risques sanitaires, est l'adaptation de l'organisation de l'entreprise adoptée par les producteurs. En effet, dans certaines zones de production, ils anticipent la survenue systématique d'algues toxiques entraînant une interdiction de commercialisation des coquillages et suspendent leur activité pendant ces périodes à risques. Cette organisation permet de réduire notamment les conséquences de ces risques sanitaires sur les emplois ${ }^{6}$.

De manière classique, les conchyliculteurs adoptent des mesures d'auto-assurance se traduisant par des réserves financières suffisantes pour pouvoir faire face à d'éventuels dommages. Depuis 2009, l'épargne de précaution est favorisée par une forme incitative de défiscalisation des

5. Paquet hygiène (CE) $n^{\circ}$ 178/2000.

6. Congés des salariés systématiquement pendant le mois où l'apparition des algues toxiques est la plus forte (juin-juillet). 
résultats annuels des entreprises. Ainsi, la Déduction pour aléas (DPA) ${ }^{7}$ permet aux conchyliculteurs soumis à un régime réel d'imposition et ayant souscrit une assurance incendie et bateau, de constituer une épargne pouvant être utilisée pour faire face à divers aléas (climatiques, sanitaires). Une enquête ${ }^{8}$ sur les entreprises ostréicoles de la baie de Bourgneuf (Loire-Atlantique) réalisée en 2007 (avant l'application de la DPA au secteur conchylicole) auprès de 43 conchyliculteurs montre que ce mécanisme incitatif à la constitution de réserves correspond à une solution d'auto-assurance souhaitée par les professionnels ( $74 \%$ des répondants se disaient prêts à y adhérer s'il était accessible).

\section{Mécanismes de transfert de risques}

Le deuxième principe de couverture des risques concerne leur transfert. Le développement récent de nouvelles relations commerciales entre différents producteurs permet à chaque partie prenante de transférer tout ou une partie des risques liés au milieu de production. Ces nouvelles formes de contractualisation concernent principalement les écloseurs et les éleveurs. La contractualisation peut prévoir une simple prestation de service où l'écloseur rémunère l'éleveur pour élever ses huîtres. L'écloseur peut également mettre à disposition d'un éleveur des poches de naissain d'huîtres, l'éleveur se rémunérant en gardant une partie du stock d'huîtres arrivées à maturité. Enfin, l'écloseur peut vendre son naissain à un éleveur et s'engage à racheter une partie du stock. Cette clause de rachat peut être interprétée comme un contrat forward sur les huîtres, le marché de gré à gré étant constitué par les écloseurs ache-

7. La Déduction pour aléas est régie par le Décret $\mathrm{n}^{\circ}$ 2009-391 du 7 avril 2009, l'article 78 de la loi $\mathrm{n}^{\circ}$ 2008-1442 du 30 décembre 2008 et les articles 72D bis et ter du Code général des impôts.

8. Enquête réalisée dans le cadre du programme de recherche Gerrico financé par la Région des Pays de la Loire (Pardo et Le Bihan., 2010). teurs de forward, et les éleveurs vendeurs de forward. L'écloseur s'engage en effet à acheter à une date fixée des huîtres pour une quantité et un prix déterminés par le contrat. Le contrat peut revêtir deux formes apportant des garanties différentes pour les deux parties :

- soit il stipule un prix fixe par kilogrammes d'huîtres et une quantité minimale rachetée ; dans ce cas, l'éleveur ne connaît pas précisément la quantité finale rachetée ; - soit il stipule un nombre de poches et un prix par poche ; le tonnage produit et le prix au kilo ne sont alors pas connus. Ce contrat permet de limiter un manque à gagner dû à un manque de rendement ou à une mortalité d'une partie du stock pour les éleveurs. Il est en revanche plus risqué pour l'écloseur.

Dans tous les cas, ces différentes contractualisations permettent de limiter l'engagement financier des écloseurs (pas de possession de parcs, pas d'achat de naissain).

Le transfert des risques s'opère également via la souscription de contrats d'assurance. Néanmoins, au-delà des contrats classiques en matière d'assurance de personne ou de dommages, l'offre d'assurance spécifique au secteur conchylicole est très peu développée. Pour les risques liés au milieu de production elle reste confidentielle. En particulier, il n'existe aucun contrat d'assurance cheptel destiné à la conchyliculture en Europe. Pourtant, selon le rapport de la FAO sur l'assurance aquacole mondiale, ce sont les pays européens qui disposent de la plus grande offre d'assurance cheptel en aquaculture (Van Anrooy et al., 2006). Mais en réalité, ces assurances sont réservées à la pisciculture. Le seul contrat existant pour les risques liés au milieu de production est une assurance multirisques agricoles proposée par le Crédit Agricole (PACIFICA), qui inclut une garantie complémentaire optionnelle appelée « report des ventes ». Elle permet d'assurer les pertes de marge brute découlant d'un arrêté préfectoral d'interdiction de 
vente due à la présence dans les coquillages de toxines répertoriées. Cette garantie est conditionnée à une durée d'interdiction au moins égale à 15 jours et l'indemnisation est calculée sur la base de la marge brute moyenne des 3 dernières années de production de l'exploitation avec une franchise de $5 \%$ du capital assuré. La souscription de ce contrat demeure aujourd'hui très faible. (Pardo, Le Bihan, 2010) montrent que moins de $8 \%$ des professionnels interrogés seraient susceptibles de souscrire une telle assurance. La principale raison invoquée est le niveau élevé de la prime et de la franchise. Pour ce risque particulier, le marché de l'assurance est clairement monopolistique, ce qui contribue à ce niveau élevé des primes. Il est intéressant de remarquer que les assureurs spécialisés dans les risques agricoles, comme GROUPAMA, ne se positionnent pas sur ce marché. Nous reviendrons sur les éléments qui freinent le développement du marché de l'assurance des risques liés au milieu de production conchylicole dans la dernière section.

Le régime d'indemnisation des catas-

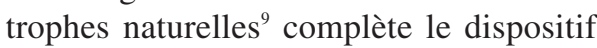
assurantiel. Ce régime est financé par un taux de surprime fixé par l'État sur les contrats d'assurance dommages aux biens. L'État garantissant la solvabilité du système via la Caisse centrale de réassurance (CCR), le déclenchement de l'indemnisation est conditionné à la reconnaissance de l'état de catastrophe naturelle au moyen d'un arrêté interministériel. Enfin, le dispositif prévoit que les assurés conservent une partie du risque par l'intermédiaire de franchises qui ne peuvent être rachetées, même par le biais d'une autre police (Monti, 2004). Ce partage de risques permet de limiter l'aléa moral. Cet instrument ne couvre qu'une partie des pertes causées par des aléas climatiques; en particulier les coquillages en élevage sont exclus de la garantie. Le régime

9. Loi n 82-600 du 13 juillet 1982. des catastrophes naturelles a été par exemple sollicité par les ostréiculteurs après la tempête Xynthia ${ }^{10}$ en février 2010 (Bersani et al., 2010).

L'indemnisation des dommages non couverts par le régime des catastrophes naturelles repose sur le Fonds national de garantie des calamités agricoles ${ }^{11}$ (FNGCA), géré par les pouvoirs publics. Entre mutualisation et solidarité, ce fonds est financé à parité par les agriculteurs et l'État. Les conchyliculteurs doivent être assurés contre l'incendie des bâtiments et la tempête et posséder une assurance risques nautiques, couvrant l'ensemble des embarcations affectées à l'exploitation. Des contributions additionnelles sur l'ensemble de ces assurances sont ainsi reversées au FNGCA. En cas de reconnaissance de la calamité agricole par l'État, les indemnités allouées par le FNGCA aux conchyliculteurs ne dépassent pas $12 \%^{12}$ des dommages subis, et ce en raison du niveau de cotisation au fonds du secteur. En effet, l'assiette de la taxe pour les exploitations conchylicoles est trop étroite et ne leur permet pas de participer suffisamment à la solidarité professionnelle (Babusiaux, 2000). Le FNGCA est intervenu après la tempête Xynthia, en complément du régime des catastrophes naturelles, pour l'indemnisation des pertes sur cheptel. De même, le FNGCA a pris en charge les indemnisations des ostréiculteurs lors des mortalités estivales qui se sont produites depuis 2008. À titre d'exemple, le montant des indemnisations versées par le FNGCA aux ostréiculteurs à la suite de ces mortalités s'élevait à 17,2 M€ en 2008 (MAP-DPMA, 2010).

10. Le montant des dommages aux biens professionnels assurés a été évalué à 157,7 M€ pour 2323 déclarations en Vendée et Charente-Maritime ; ces dommages correspondant majoritairement à des dégâts advenus aux exploitations ostréicoles.

11. Loi n 64-706 du 10 juillet 1964.

12. Article 49 de la loi de finances 71-1061 du 29/12/1971. 


\section{Solidarité}

Compte tenu du faible développement des assurances des risques sanitaires, l'intervention publique joue un rôle important dans la gestion des risques conchylicoles, à travers le FNGCA et le fonds catastrophes naturelles mais également par des mécanismes de solidarité. La solidarité intervient à différents échelons territoriaux (département, région, État) et est déclenchée au cas par cas selon la gravité des événements. Le Gouvernement a mis en place un tel plan de soutien exceptionnel aux conchyliculteurs et pisciculteurs, à la suite de la tempête Xynthia ${ }^{13}$. Le montant total de l'aide s'est élevé à 23 millions d'euros représentant $32,8 \%$ du dommage total et se déclinait en diverses mesures : aide au remplacement du matériel, allègement des charges financières, report de paiement de charges sociales.

Au niveau européen, le Fonds européen pour la pêche ${ }^{14}$ (FEP) finance des mesures de protection sanitaire des cheptels ou de protection de la santé humaine. Via l'article 31, il peut contribuer aux indemnités d'arrêt temporaire des activités de récolte et de commercialisation des mollusques d'élevage, à des fins de protection de la santé publique. L'indemnisation est déclenchée à l'issue d'une interdiction de commercialisation de plus de quatre mois consécutifs, ou lorsque le préjudice subi représente plus de $35 \%$ du chiffre d'affaires annuel de l'entreprise. Les seuils de déclenchement des contributions sont donc très élevés et correspondent à des évènements extrêmes de faible probabilité. Le FEP n'a d'ailleurs encore jamais été activé pour des fermetures sanitaires. Le FEP peut également contribuer au financement du contrôle et de l'éradication des maladies en aquaculture (article 32).

13. Commission européenne, C (2010) 2642, Aide d'État N 119/2010 - France - Plan de soutien exceptionnel aux conchyliculteurs et pisciculteurs des départements touchés par la tempête Xynthia dans la nuit du 27 au 28 février 2010.

14. Règlement (CE) $\mathrm{N}^{\circ} 1198 / 2006$ du Conseil du 27 juillet 2006.
Un dernier mécanisme de solidarité existe au niveau international, les Fonds internationaux d'indemnisation pour les dommages dus à la pollution par les hydrocarbures (FIPOL), reposant sur le principe pollueurpayeur. Financés par les États importateurs de pétrole et les propriétaires de navires pétroliers, les FIPOL interviennent en complément de l'assureur du navire, lorsque le montant du dommage par pollution excède le montant de la réparation fixée par la convention de 1992 sur la responsabilité civile. Sont couverts par les fonds, toute perte ou tout dommage causé par une contamination. À la suite des naufrages pétroliers Erika (1999) et Prestige (2002), les conchyliculteurs ont eu recours à ces fonds pour indemniser les préjudices qu'ils ont subis.

Les systèmes de solidarité présentent trois principales limites. Les délais d'indemnisation sont parfois très longs, créant des décalages de trésorerie pénalisants. Les systèmes permettent de compenser en partie seulement les dommages supportés (Le Bihan et al., 2008) et les coûts administratifs sont importants (Mahul, 1998).

\section{Limites de la couverture des risques conchylicoles}

Les outils de couverture des risques liés au milieu de production conchylicole présentés dans la précédente section, apparaissent aujourd'hui insuffisants au regard des événements récents. Dans un contexte d'augmentation des sinistres (augmentation du nombre de fermetures administratives (Belin, op. cit.), mortalités estivales anormales depuis 2008 (Cochennec-Laureau, Baud, op. cit.) et d'affermissement des normes réglementaires, les conchyliculteurs expriment un besoin accru de couverture des risques. Ces exigences de protection se heurtent à un certain nombre de limites concernant les possibilités d'adaptation ou de mise en œuvre de nouveaux mécanismes de couverture. 


\section{Développement limité des mesures de prévention et auto-assurance}

L'étendue des mesures d'auto-protection et d'auto-assurance des professionnels est en effet limitée. En termes d'auto-protection, la diversification des espèces élevées se heurte aux distances entre concessions et établissement conchylicole. Dépendants de la localisation de leurs parcs, les exploitants sont généralement spécialisés : $70 \%$ dans un seul type de coquillage (MAPDPMA, 2009) ce qui limite les possibilités d'atténuation lorsqu'un préjudice est subi sur une espèce. Enfin, les possibilités de diversification des entreprises conchylicoles sont limitées compte tenu d'une allocation stable, voire en diminution des surfaces d'élevage conchylicoles sur le DPM. Aussi, les professionnels ne peuvent se diversifier qu'en accédant à des parcs déjà concédés et cédés, la plupart du temps, lors d'une cessation d'activité. Les indemnités de cession à verser pour en disposer, l'augmentation du coût d'exploitation qui en découle et les investissements supplémentaires nécessaires à leur exploitation sont autant d'éléments qui ne permettent pas aux petites entreprises familiales d'y accéder facilement.

Pour faire face à la baisse de rendement des parcs, les conchyliculteurs opèrent des transferts réguliers de coquillages entre bassins de production, et plus particulièrement des huîtres en fonction de leur maturité. Or, la recherche d'une allocation optimale des stocks pour une meilleure productivité peut conduire à une exposition plus grande à certains risques climatiques et sanitaires. Les pratiques et usages des professionnels, à travers les différentes manipulations, les densités et les transferts intra ou inter bassins des cheptels, peuvent ainsi être à l'origine d'une prolifération plus rapide des épizooties. Aujourd'hui, l'une des limites de développement des mesures d'auto-protection des professionnels tient à la méconnaissance des impacts potentiels de ces pratiques sur le niveau des pertes.
Le rapatriement et la sauvegarde des coquillages dans des bassins insubmersibles ou dans des zones protégées pourraient constituer une mesure d'auto-assurance contre les risques sanitaires et ceux de la pollution par hydrocarbure. Cependant, cette solution est très difficile à mettre en œuvre, sauf pour des volumes très restreints et pour une durée limitée. Cela suppose, des capacités de stockage suffisantes sur estran, dans des zones non impactées, ou en bassins insubmersibles ; capacités dont ne dispose pas la majorité des professionnels. Quant aux procédés de détoxication des coquillages, ils nécessitent des dispositifs de traitement de l'eau et d'apports en nutriments pour les cheptels. Aujourd'hui, ces procédés ne permettent pas d'éliminer l'ensemble des algues toxiques et représentent un coût important pour les entreprises (Haure et al., 2011). Sur la côte atlantique et la Manche, le rapatriement des mollusques suppose une disponibilité de marées de vives eaux.

Il faut également signaler que certains outils permettant d'atténuer les coûts des sinistres sont à la disposition des professionnels mais ils y ont peu recours. La mise sous forme sociétaire de l'entreprise permet de protéger le patrimoine privé vis-à-vis des créanciers. Or, $73 \%$ des entreprises conchylicoles sont des entreprises familiales (MAP-DPMA, 2009). Les avantages ne sont pas perçus comme supérieurs aux coûts de la mise sous forme sociétaire de l'entreprise. Plus généralement, on peut constater le fort individualisme des conchyliculteurs qui ne les pousse pas à s'associer pour partager les risques.

\section{2. Éléments d'inassurabilité}

Comme nous l'avons souligné, il n'existe pratiquement pas de contrats d'assurance sur les principaux besoins des professionnels à savoir les risques liés au milieu de production. Cette absence de couverture assurantielle interroge sur l'assurabilité de ces risques et sur les possibilités d'améliorer l'offre de couverture. 
Un risque est dit assurable s'il satisfait les critères suivants (Berliner, 1982 ; Couture, op. cit.) : il revêt un caractère réellement aléatoire (date de survenue d'un sinistre non prévisible) ; le risque est bien défini ; la loi des grands nombres doit fonctionner (un grand nombre de risques individuels homogènes et indépendants existent) ; le risque est quantifiable (probabilités calculables, montant des pertes évaluable et non manipulable); le risque n'est pas de type catastrophique pour l'assureur ; la prime d'assurance est économiquement acceptable. Gollier (op. cit.) suggère, lui, qu'un risque est inassurable dès lors qu'aucun transfert de risque mutuellement avantageux ne peut être exploité par le consommateur et le fournisseur d'assurance. Gollier (2005) précise que les sources d'inassurabilité sont multiples même si elles aboutissent toutes à la même conclusion : si certains risques sont inassurables, c'est parce que les coûts directs et indirects induits par l'assurance excèdent les coûts de l'inassurabilité. Les risques liés au milieu de production conchylicole ne satisfont pas toutes les hypothèses standard d'assurabilité, ce qui peut expliquer le faible nombre de contrats dans ce secteur. Une compréhension des principaux facteurs entrainant la violation de ces hypothèses, est nécessaire pour envisager une amélioration de la couverture de ces risques.

\section{Définition délicate des risques sanitaires} Comme nous l'avons évoqué précédemment, les causes des mortalités estivales anormales des huîtres, constatées depuis 2008 sur toutes les façades littorales françaises, ne sont pas connues avec précision ${ }^{15}$. Conjonction de phénomènes climatiques et épidémiologiques, influence des transferts de coquillages dans la propagation du phénomène, certaines de ces hypothèses commencent à peine à être validées, avec précaution, par les scientifiques. De manière générale,

15. http://wwz.ifremer.fr/institut/actualites/ mortalites_d_huitres. la mortalité des coquillages peut être imputée à plusieurs facteurs et de ce fait, c'est un risque très mal défini qui entraîne la réticence des assureurs. Sur ce point, seules les avancées des recherches scientifiques peuvent améliorer la définition de ces risques et permettre de surmonter cette difficulté.

\section{Étroitesse du secteur et coûts de transaction élevés}

Le montant des coûts de transaction peut être source d'inassurabilité partielle en générant des primes d'assurance trop élevées (Gollier, 1996). En conchyliculture, le caractère inhabituel des risques en jeu et l'étroitesse du secteur d'activité peuvent conduire à des frais de mise en œuvre des contrats élevés et à des primes qui ne sont pas économiquement acceptables. Les assureurs cherchent à placer les risques dans un groupe homogène mais indépendant qui leur permet de répartir les risques et de bénéficier de la loi des grands nombres. En supposant que l'ensemble des conchyliculteurs souscrit à un contrat d'assurance spécifique contre un risque lié au milieu de production, la mutualisation ne permettrait pas forcément de proposer un contrat optimal. La concentration du secteur ces dernières années ainsi que les phénomènes de mortalités estivales de 2008 à 2010 fragilisent les entreprises et ne rendent pas possible un élargissement du nombre de cotisants.

Forte corrélation des risques individuels La concentration géographique des activités ajoute une difficulté en remettant partiellement en cause le caractère indépendant des risques (Pardo et al., 2008). Il faut noter que les transferts opérés entre les bassins à plusieurs stades du cycle de production accentuent ce phénomène. Ainsi, pour les risques sanitaires, une forte corrélation des risques individuels existe. Le risque systémique est donc important comme l'illustrent les mortalités estivales de 2008 à 2010, enregistrées sur l'ensemble des bassins français. Ce risque de mortalité généralisée, 
voire de disparition de l'espèce (cas de l'huître plate, puis de l'huître portugaise respectivement dans les années 1920 et 1970) peut conduire à des sinistres très importants mettant en péril l'équilibre financier des assureurs, et accroît la difficulté à mobiliser des réassureurs sur ces questions (Besème et al., 2009). La présence de risque systémique peut en effet conduire à la défaillance du marché de l'assurance dans le secteur considéré. Miranda et Glauber (1997) ont étudié le marché de l'assurance récolte privée aux USA, sur lequel les agents sont exposés au risque climatique. Les auteurs montrent que les risques individuels présentant une très forte corrélation, les portefeuilles des assureurs récolte sont 20 à 50 fois plus risqués qu'en l'absence de cette corrélation.

\section{Ambiguïté}

Un quatrième facteur d'inassurabilité relève de l'ambiguïté due à l'absence de probabilités objectives pour décrire certains risques sanitaires et climatiques. La décision en présence d'ambiguïté a fait l'objet de nombreux travaux théoriques (Ellsberg, 1961 ; Ghirardato, Marinacci, 2002). Gilboa et Schmeidler (1989) ont donné un cadre théorique au concept d'aversion pour l'ambiguiité. Dans le cadre de l'assurance, l'aversion pour l'ambiguiité va avoir tendance à augmenter la demande (Brunette et al., 2009 ; Camerer, Weber, 1992 ; Hogarth, Kunreuther, 1989, 1992). En revanche, l'aversion pour l'ambiguïté des assureurs augmente le prix des contrats (Kunreuther $e t$ al., 1995 ; Cabantous, 2007) et représente un facteur d'inassurabilité, en particulier si seul l'assureur est averse à l'ambiguïté ou s'il l'est plus que ne l'est l'assuré (Gollier, 1996).

En conchyliculture, les risques sanitaires présentent une forte ambiguïté car les aléas naturels sont multiples et mal connus et sont soumis à l'incertitude scientifique. Par exemple, pour les moules, le lien de causalité entre présence d'algues toxiques et contamination des coquillages n'est pas établi, et le processus de contamination reste mal connu (Haure et al., op. cit.). Ainsi, la probabilité de contamination et, par conséquent, d'interdiction de commercialisation de ces coquillages demeure imprécise. Par ailleurs, un environnement réglementaire en évolution tend à augmenter l'aversion pour l'ambiguïté des assureurs. La probabilité d'occurrence peut en effet être modifiée lors de décisions administratives qui peuvent accroître de façon importante et non maîtrisée les dommages indemnisables par l'assureur (arrêt des transferts lors d'épisodes de toxicité ou de mortalité, etc.).

Les risques climatiques et les risques de pollution présentent également de l'ambiguïté de part la rareté des phénomènes (tempêtes et submersions marines en particulier, marées noires) et la difficulté à les prévoir. Le manque d'observations ne permet pas aujourd'hui d'établir une estimation fiable des probabilités de ces évènements.

Par ailleurs, l'imprécision actuelle sur l'ampleur des conséquences du changement climatique sur les risques de tempête, submersion marine et autres aléas naturels, accroît l'ambiguiité des risques climatiques et sanitaires.

\section{Quantification des dommages et aléa moral} expost

Malgré l'obligation de déclaration de production $^{16}$, celle-ci n'est pas entièrement respectée par les professionnels alors que les contrôles et sanctions ne sont pas toujours mis en œuvre par les autorités. Besème et al. (op. cit.) précisent que le cheptel présent sur les parcs est difficilement quantifiable. L'expertise sur place est complexe et coûteuse, et l'absence de barèmes officiels de rendements par zone, comme cela est le cas en agriculture, rend difficile une évaluation forfaitaire fiable. Enfin, tous les ostréiculteurs ne sont pas imposables au réel ce qui rend difficile l'évaluation des pertes

16. Décret du 22 mars 1983. 
lors d'un sinistre, à partir de documents comptables. Comme le montre Le Bihan (2003) le secteur conchylicole souffre d'un déficit en statistiques économiques, financières et sociales lequel ne permet pas d'établir le montant des préjudices réellement subis. Si, les durées de phycotoxicité dans les coquillages du littoral français sont connues, en revanche, l'évaluation de l'impact économique de ces blooms ${ }^{17}$ a fait l'objet de peu d'études. Stolte et al. (2001) analysent l'impact socio-économique des blooms de micro algues toxiques dans les pays de l'Union européenne. L'estimation des dommages est comprise entre 280 et 1252 millions d'euros par an, entre 1989 et 1998, dont 56 à 219 millions d'euros concernent les pertes des producteurs et consommateurs de moules. Ce type d'évaluation se révèle très difficile compte tenu de conséquences directes et induites multiples et de la possibilité d'échelonnement des effets. Il existe ainsi un risque moral ex post sur les déclarations de sinistres. Il ne s'agit pas seulement de risque de fraude mais plus généralement du problème lié à l'inobservabilité des pertes par l'assureur lors d'un sinistre.

\section{Intervention publique et assurance}

Le dernier facteur d'inassurabilité concerne l'adéquation entre l'offre de contrat d'assurance et le niveau de prix acceptable pour les assurés. Blanc (2003) souligne que le niveau de prime demandé doit être inférieur au consentement à payer. Un consentement à payer insuffisant peut résulter de différents facteurs tels qu'une aversion au risque insuffisante des agents ou l'existence de dispositifs d'indemnisations publics gratuits concurrents de l'assurance.

Dans le cas de la conchyliculture, si les assureurs avaient les moyens de surmonter les difficultés liées à la mauvaise connaissance des risques rattachés au milieu de production, le soutien financier systéma-

17. Que l'on peut traduire par prolifération. tique des pouvoirs publics en cas de calamités agricoles pourrait être un frein au développement de ce marché. D'un point de vue théorique, il est en effet admis que l'existence d'aides publiques crée un problème de risque moral ex ante, incitant les agents à ne pas prendre des mesures de prévention nécessaires (Brunette, Couture, 2008 ; Latruffe, Picard, 2005). Une évolution des fonds de solidarité serait alors nécessaire pour permettre le développement de contrats d'assurance privée. Les expérimentations en assurances multirisques climatiques pour certaines cultures, fruitières notamment, laissent entrevoir des possibilités d'adaptation (participation de l'État à la prime d'assurance, pas de recours au FNGCA pour les cultures assurables, GROUPAMA assurance CLIMATS).

\section{Conclusion}

Le secteur conchylicole doit faire face à de nombreux risques, souvent très spécifiques au regard du milieu dans lequel s'exercent ces activités. Entièrement tributaires des aléas climatiques et sanitaires, les possibilités de prévention et d'autoassurance sont limitées. La non-appropriation du foncier et la méconnaissance des impacts potentiels des pratiques et usages sur le niveau des préjudices ne facilitent pas la gestion des risques. Le nombre restreint de produits assurantiels s'explique par la présence de facteurs d'inassurabilité. L'ambiguïté et le risque moral ex post restent les plus difficiles à surmonter et nécessitent un travail en amont impliquant les professionnels, les scientifiques et les assureurs. Afin d'améliorer la couverture des risques liés au milieu de production, une solution serait la mise en place d'un fonds de garantie professionnel, adossé à l'État, pour la prise en charge des risques sanitaires. Les assurances privées pourraient plus facilement se positionner sur les risques de tempête ; la difficulté principale résidant dans la quantification du risque. Une 
participation de l'État à la prime d'assurance pourrait également être envisagée. La connaissance fine du cheptel et des pertes potentielles semblent être un préalable à tout travail prospectif.
Les auteures remercient la Région des Pays de la Loire pour le financement de ce travail dans le cadre du programme Gerrico et également les deux rapporteurs anonymes pour leurs remarques constructives.

\section{RÉFÉRENCES BIBLIOGRAPHIQUES}

Arnason R. (2007). Climate change and fisheries: assessing the economic impact in Iceland and Greenland. Natural Resource Modeling, vol. 20, $\mathrm{n}^{\circ}$ 2, p. 163-197.

Babusiaux C. (2000). L'assurance récolte et la protection contre les risques en agriculture. Rapport interministériel. Octobre, $288 \mathrm{p}$.

Beach R.,Viator C. L. (2008). The economics of aquaculture insurance : an overview of the U.S. pilot insurance program for cultivated clams. Aquaculture Economics \& Management, vol. $12, \mathrm{n}^{\circ} 1$, p. 25 38.

Belin C. (2005). Bilan sur 20 ans des interdictions administratives de vente et de ramassage des coquillages, pour présence de phycotoxines, sur le littoral français (1984-2003). Ifremer, $84 \mathrm{p}$.

Berliner B. (1982). Limits of insurability of risks. Prentice Hall in Englewood Cliffs, N.-J., 118 p.

Bersani C., Gérard F., Gondran O., Helias A., Martin X., Puech P., Dumas P., Rouzeau M., Fleury B., Greff M., Bougère R., Trepos Y. (2010). Tempête Xynthia. Retour d'expérience, évaluation et propositions d'actions. Tome 1, rapport interministériel, mai, $191 \mathrm{p}$.

Besème J.-L., Defrance D., Suche J.-M. Legendre H. (2009). Procédures de couverture des risques conchylicoles. Rapport interministériel, 83 p.

Blanc C. (2003). La gestion des risques en agriculture. Modèle offre-demande d'assurance récolte. Ministère de l'économie, des finances et de l'industrie, direction de la Prévision, $40 \mathrm{p}$.
Bommelaer O, Devaux J. (2011). Coûts des principales pollutions agricoles de l'eau. Études et Documents $\mathrm{n}^{\circ} 52$, Commissariat général au développement durable, $32 \mathrm{p}$.

Brunette M., Couture S. (2008). Assurance et activités de réduction des risques en foresterie : une approche théorique. Review of Agricultural and Environmental Studies, vol. 86, $\mathrm{n}^{\circ}$ 1, p. 55-78. Brunette M., Cabantous L., Couture S., Stenger A. (2009). Assurance, intervention publique et ambiguïté : une étude expérimentale auprès de propriétaires forestiers privés. Economie \& prévision, vol. 190-191, n 4-5, p. 123-134.

Cabantous L. (2007). Ambiguity Aversion in the Field of Insurance: Insurers' Attitude to Imprecise and Conflicting Probability Estimates. Theory and Decision, vol. 62, n ${ }^{\circ} 3$, p. 219-240.

Camerer C., Weber M. (1992). Recent developments in modeling preferences: Uncertainty and ambiguity. Journal of Risk and Uncertainty, vol. 5, $\mathrm{n}^{\circ}$ 4, p. 325-370.

Cochennec-Laureau N., Baud J.-P. (2011). Bilan des surmortalités des huîtres creuses Crassostrea gigas depuis 2008. Bulletin épidémiologique, $\mathrm{n}^{\circ} 42$, p. 2-5.

Collart-Dutilleul F. (2003). Le principe de précaution dans le règlement communautaire du 28 janvier 2002. Giuffre (éd.), $239 \mathrm{p}$.

Couture S. (2009). Analyse du comportement individuel et collectif des professionnels forestiers face aux risques, réflexion sur la dimension assurantielle. Innovations Agronomiques, ${ }^{\circ} 6$, p. 73-85. 
Ehrlich I., Becker G.-S. (1972). Market insurance, self-insurance and self-protection. The Journal of Political Economy, vol. 80, n 4, p. 623-648.

Ellsberg D. (1961). Risk, Ambiguity, and the Savage xiom. Quarterly Journal of Economics, vol. 75, n 4, p. 643-669.

Gervasoni E., Perignon A., Sourisseau E., Rey-Valette H., Lagarde F., Perez J., Yimam E., Feldman N. (2011). Monographie - La conchyliculture en Méditerranée, $29 \mathrm{p}$.

Ghirardato P., Marinacci M. (2002). Ambiguity Made Precise: A Comparative Foundation. Journal of Economic Theory, vol. $102, \mathrm{n}^{\circ} 2$, p. 251-289.

Gilboa I., Schmeidler D. (1989). Maxmin expected utility with non-unique prior. Journal of Mathematical Economics, vol. 18, $\mathrm{n}^{\circ}$ 2, p. 141-153.

Gitay H., Suárez A., Watson R.-T. (2002). Les changements climatiques et la biodiversité. Document technique V du GIEC, CBD, WMO, PNUE, 89 p.

Gollier C. (1996). Vers une théorie économique des limites de l'assurabilité. Revue d'Économie Financière, no 37, p. 59-79.

Gollier C. (2005). Coûts de l'inassurabilité et coûts de l'assurance. Revue d'Économie Financière, vol. 80, $\mathrm{n}^{\circ} 3,14 \mathrm{p}$.

Hallegatte S., Hourcade J., Dumas P. (2007). Why economic dynamics matter in assessing climate change damages: Illustration on extreme events. Ecological Economics, vol. 62, $\mathrm{n}^{\circ}$ 2, p. 330-340.

Hardaker J.-B., Huirne R.-B.-M., Anderson J.-R., Lien G. (2004). Coping with risk in agriculture. Wallingford, Oxfordshire, CABI Publishing, 332 p.

Haure J., Hussenot J., Buzin F., Lassus P., Marcaillou-Le Baut C., Mondeguer F., Sechet V., Royer F., Amzil Z., Cardinal M., Belin C., LE Grel L., Le Bihan V. (2011). COMSAUMOL (maintien de la COMmercialisation par la SAUvegarde et la détoxication des MOLlusques), Université de Nantes. IFREMER, $92 \mathrm{p}$.
Hogarth R. M., Kunreuther H. (1989). Risk, ambiguity, and insurance. Journal of Risk and Uncertainty, vol. 2, $\mathrm{n}^{\circ}$ 1, p. 5-35.

Hogarth R. M., Kunreuther H. (1992). How Does Ambiguity Affect Insurance Decision? Contributions to Insurance Economics, George Dionne (ed.), 27 p.

Jaffee D.-M., Russell T. (1997). Catastrophe insurance, Capital Markets and Uninsurable Risks. The Journal of Risk and Insurance, $\mathrm{n}^{\circ} 64$, p. 205-230.

Kunreuther H., Meszaros J., Hogarth R.-M., Spranca M. (1995). Ambiguity and underwriter decision processes. Journal of Economic Behavior \& Organization, vol. 26, n ${ }^{\circ} 3$, p. 337-352.

Latruffe L., Picard P. (2005). Assurance des catastrophes naturelles : faut-il choisir entre prévention et solidarité ? Annales d'économie et de Statistique, $\mathrm{n}^{\circ} 78$, p. 33-56.

Le Bihan V. (2003). Diagnostic de l'installation des jeunes dans le domaine des cultures marines sur la facade Aglia. Rapport pour l'Observatoire des Pêches et des Cultures Marines de l'Aglia, LEN, Université de Nantes, 62 p.

Le Bihan V. (2006). La transmission des entreprises conchylicoles sur la façade Atlantique : un secteur en mutation marqué par un renouvellement difficile des entreprises. Actes du colloque «Pour une exploitation durable des ressources vivantes de la mer et du littoral ». Guillaume J. (coord.), Rennes, LETG UMR 6554 CNRS, p. 217-228.

Le Bihan V., Pardo S., Perraudeau Y. (2007). La gestion globale des risques en conchyliculture. Rapport pour l'Observatoire des pêches et des cultures marines du golfe de Gascogne, AGLIA, $41 \mathrm{p}$.

Le Bihan V., Le Grel L., Perraudeau Y. (2008). Aquaculture. In Guillotreau P. (ed.), «Mare Economicum », P.U.R., p. 139-191.

MAP-DPMA. (2009). Enquête Aquaculture $2007.6 \mathrm{p}$. 
MAP-DPMA. (2010). Les concours publics aux pêches maritimes et aux cultures marines en 2008. 12 p.

Mahul O. (1998). Vers une redéfinition du rôle de l'assurance agricole dans la gestion des risques sur récoltes. Cahiers d'économie et sociologie rurales, $\mathrm{n}^{\circ} 49$, p. 33-58.

Maurer D., Comps M., His E. (1986). Caractéristiques des mortalités estivales de l'huître Crassostrea Gigas dans le bassin d'Arcachon. Haliotis, n 15, p. 309-317.

Miranda M. J., Glauber J. W. (1997). Systematic risk, reinsurance, and the failure of crop. American Journal of Agricultural Economics, vol. 79, n ${ }^{\circ}$, p. 206-215.

Mongruel R., Perez Agundez J.-A., Girard S. (2006). Droits à produire transférables : Le marché des concessions conchylicoles et ses effets non désirés. Économie rurale, $\mathrm{n}^{\circ} 306$, p. 23-38.

Monti A. (2004). Assurance et risques environnementaux : une analyse comparative du rôle de l'assurance dans la gestion des risques liés à l'environnement. OCDE, $101 \mathrm{p}$.

Pardo S., Perraudeau Y., Rautureau N. (2008). Apports de la finance au secteur maritime. In Guillotreau P. (ed.), « Mare Economicum », P.U.R., p. 241-279.

Pardo S., Le Bihan V. (2010). Gestion globale des risques. Gestion globale des ressources marines et des risques dans les espaces côtiers. IFREMER, Université de Nantes. Recherche financée par la région des Pays de la Loire. Rapport de recherche, vol. 2, p. 57-65.

Pörtner H.-O. (2008). Ecosystem effects of ocean acidification in times of ocean warming: a physiologist's view. Marine Ecology Progress Series, $n^{\circ}$ 373, p. 203-217. Samain J.-F., McCombie H. (2007). Mortalités estivales de l'huître creuse Crassostrea gigas, Défi Morest. Quae, $331 \mathrm{p}$.

Stolte W., Scatasta S., Graneli E., Weikard H.-P., Van Ierland E. (2001). ECOHARM: The socio-economic impact of harmful algal blooms in european marine waters. Department of Biology and Environmental Science, Kalmar University, Sweden \& Environmental Economics and Natural Resources Group, Wageningen University, $100 \mathrm{p}$.

Van Anrooy R., Secretan P. A. D., Lou Y., Richard R., Upare M. (2006). Review of the current state of world aquaculture insurance. FAO Rome, $107 \mathrm{p}$.

Vigo J. (2001). La mer et le littoral en Bretagne. Pour une ambition régionale. Conseil Economique et Social de Bretagne, $232 \mathrm{p}$.

Vézina A. F., Hoegh-Guldberg O. (2008). Effects of ocean acidification on marine ecosystems: introduction. Marine Ecology Progress Series, n³73, p. 199-201.

Zeckhauser R. (1995). Insurance and Catastrophes. Geneva paper on Risk and Insurance Theory, $\mathrm{n}^{\circ} 20$, p. 157-175. 